\title{
Heterozygous TREM2 mutations in frontotemporal dementia
}

\author{
Barbara Borroni ${ }^{\mathrm{a}, *}$, Francesca Ferrari ${ }^{\mathrm{a}}$, Daniela Galimberti ${ }^{\mathrm{b}}$, Benedetta Nacmias ${ }^{\mathrm{c}}$, Cinzia Barone ${ }^{\mathrm{b}}$, \\ Silvia Bagnoli ${ }^{c}$, Chiara Fenoglio ${ }^{\mathrm{b}}$, Irene Piaceri ${ }^{\mathrm{c}}$, Silvana Archetti ${ }^{\mathrm{d}}$, Cristian Bonvicini $^{\mathrm{e}}$, \\ Massimo Gennarelli ${ }^{\mathrm{e}}$, Marinella Turla ${ }^{\mathrm{f}}$, Elio Scarpini ${ }^{\mathrm{b}}$, Sandro Sorbi ${ }^{\mathrm{c}}$, Alessandro Padovani ${ }^{\mathrm{a}}$ \\ ${ }^{a}$ Center of Neurodegenerative Disorders, Neurology Unit, University of Brescia, Brescia, Italy \\ ${ }^{\mathrm{b}}$ Neurology Unit, Department of Pathophysiology and Transplantation, University of Milan, Fondazione Cà Granda, IRCCS Ospedale Maggiore Policlinico, Milan, Italy \\ ${ }^{\mathrm{c}}$ Department of Neuroscience, Psychology, Drug Research and Child Health, University of Florence, Florence, Italy \\ d III Laboratory of Analysis, Brescia Hospital, Brescia, Italy \\ e Istituto di Ricerca e Cura a Carattere Scientifico Fatebenefratelli, Brescia, Italy \\ ${ }^{\mathrm{f}}$ Neurology Unit, ValleCamonica Hospital, Brescia, Italy
}

\section{Introduction}

Frontotemporal dementia (FTD) is a heterogeneous neurodegenerative disorder characterized by behavioral disturbances, language impairment, and deficits in executive functions (Seelaar et al., 2011). On the basis of presenting clinical symptoms, the behavioral variant of FTD (bvFTD), agrammatic variant of primary progressive aphasia, and semantic variant of primary progressive aphasia (svPPA) represent the most common phenotypes (Gorno-Tempini et al., 2011; Rascovsky et al., 2011).

FTD has a strong genetic background, as suggested by positive family history in almost $40 \%$ of patients, and several monogenic forms of FTD have been identified. Mutations within microtubule-associated protein tau (MAPT) and granulin (GRN) genes along with repeat expansion of C9orf72 gene represent the most frequent causes of autosomal dominant inherited disorder

\footnotetext{
* Corresponding author at: Department of Neurology, University of Brescia, Piazza Spedali Civili 1-25125 Brescia, Italy. Tel.: +39 030 3995632; fax: +39 0303995027. E-mail address: bborroni@inwind.it (B. Borroni).
}

(Rademakers et al., 2012; Rohrer et al., 2011). Despite a giant step forward in the knowledge of genetic bases of FTD, several cases even with positive family history remain of unknown pathogenesis. The identification of new genetic predisposing factors, even rare variants, is key to further elucidating the pathogenetic mechanisms leading to frontotemporal brain damage.

Homozygous loss-of-function mutations in TREM2, encoding the triggering receptor expressed on myeloid cells 2 protein, have been previously associated with an autosomal recessive form of early-onset dementia presenting with bone cysts and consequent fractures called polycystic lipomembranous osteodysplasia with sclerosing leukoencephalopathy, or Nasu-Hakola disease (Paloneva et al., 2000, 2002). Interestingly, it has been reported that homozygous TREM2 exon 2 mutations may resemble FTD clinical phenotype without any bone-associated symptoms (Guerreiro et al., 2013a). Nevertheless, heterozygosis of TREM2 exon 2 mutations also has a role in neurodegenerative disorders because it has recently been associated with increased risk of late-onset Alzheimer's disease (AD) (Guerreiro et al., 2013b; Jonsson et al., 2013). 
IaDie I

TREM2 exon2 genetic variations in the screened population

\begin{tabular}{|c|c|c|c|c|c|c|c|c|c|c|}
\hline TREM2 mutation & SNP & $\begin{array}{l}\text { FTD \% } \\
\text { (n) }\end{array}$ & $\begin{array}{l}\mathrm{HC} \% \\
(\mathrm{n})\end{array}$ & $\begin{array}{l}\mathrm{AD} \% \\
\text { (n) }\end{array}$ & $\begin{array}{l}p \\
\text { FTD vs. HC }\end{array}$ & $p$ FTD vs. AD & $\begin{array}{l}p \\
\text { AD vs. HC }\end{array}$ & $\begin{array}{l}\text { OR }(95 \% \mathrm{CI}) \\
\text { FTD vs. HC }\end{array}$ & $\begin{array}{l}\text { OR }(95 \% \mathrm{CI}) \\
\text { FTD vs. AD }\end{array}$ & $\begin{array}{l}\text { OR }(95 \% \mathrm{CI}) \\
\text { AD vs. HC }\end{array}$ \\
\hline Overall & - & $4.0(14)$ & $1.0(5)$ & $2.6(5)$ & 0.005 & 0.393 & 0.126 & $3.97(1.42-11.12)$ & $1.57(0.56-4.41)$ & $2.53(0.72-8.85)$ \\
\hline$Q_{33 X^{\mathrm{a}}}$ & rs104894002 & $0.9(3)$ & $0(0)$ & $1.5(3)$ & 0.042 & 0.457 & 0.023 & NA & $0.54(0.11-2.74)$ & NA \\
\hline $\mathrm{R} 47 \mathrm{H}$ & rs75932628 & $0.3(1)$ & $0(0)$ & $0(0)$ & 0.241 & 0.457 & - & NA & NA & $\mathrm{NA}$ \\
\hline $\mathrm{R} 62 \mathrm{H}$ & rs143332484 & $0.9(3)$ & $0.4(2)$ & $0.5(1)$ & 0.221 & 0.466 & 0.637 & $2.77(0.50-15.21)$ & $2.22(0.24-20.00)$ & $1.30(0.11-13.85)$ \\
\hline $\mathrm{T}_{66} \mathrm{M}^{\mathrm{a}}$ & rs201258663 & $0.3(1)$ & $0(0)$ & $0(0)$ & 0.241 & 0.457 & - & NA & NA & NA \\
\hline D87N & rs142232675 & $0.6(2)$ & $0.2(1)$ & $0(0)$ & 0.388 & 0.293 & 0.714 & $2.76(0.29-30.56)$ & NA & NA \\
\hline T96K & rs2234253 & $0.9(3)$ & $0.2(1)$ & $0(0)$ & 0.182 & 0.197 & 0.714 & $4.15(0.43-40.08)$ & NA & $\mathrm{NA}$ \\
\hline R98W & rs147564421 & $0(0)$ & $0.2(1)$ & $0.5(1)$ & 0.393 & 0.178 & 0.491 & NA & NA & $2.5(0.15-40.21)$ \\
\hline S116C & - & $0.3(1)$ & $0(0)$ & $0(0)$ & 0.241 & 0.457 & - & NA & NA & $\mathrm{NA}$ \\
\hline
\end{tabular}

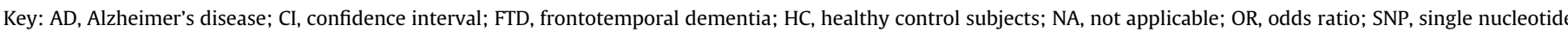
polymorphism.

a Mutation causative of Nasu-Hakola disease in homozygous state.

These observations defined the aim of the present study, and we hypothesized that variations within TREM2 gene might be associated with a broader clinical spectrum and might predispose to FTD phenotype in heterozygous state. We evaluated genetic variations within TREM2 exon 2 gene in a large cohort of patients with FTD, compared with healthy control subjects and a group of patients with AD.

\section{Methods}

\subsection{Subjects}

Patients fulfilling revised criteria for FTD (Gorno-Tempini et al., 2011; Rascovsky et al., 2011) were consecutively recruited from the Center for Aging Brain and Neurodegenerative Disorders, University of Brescia, the Neurology Unit, University of Florence, and the Neurology Unit, University of Milan, Italy.

All subjects underwent a somatic and neurologic evaluation, routine laboratory examination, and brain structural imaging study. A standardized neuropsychological assessment including global cognitive evaluation and a standardized neuropsychological test battery for investigating the main cognitive domains was performed. The diagnostic assessment included a review of full medical history, a semistructured neurologic examination, and a complete mental status evaluation. Patients considered to have a positive family history were those who had a first-degree relative with dementia, parkinsonism, or motor neuron disease. No patients belonging to the same family were included. A venous blood sample was drawn from each patient for TREM2 exon 2 sequencing.

Patients with MAPT or GRN mutations and with repeat expansion of C9orf72 gene were excluded (Borroni et al., 2011; Galimberti et al., 2013). Moreover, a control group similar in age and gender composition was recruited in the same Italian area from which the patients were drawn and was sequenced for TREM2 exon 2 genetic variations. Two subgroups were considered for comparisons, that is, subjects found to be cognitively intact, following medical history, presence of comorbidities, and neuropsychological examination (healthy controls [HCs]), and patients fulfilling clinical diagnosis of AD according to current criteria (McKhann et al., 1984).

Informed consent was obtained for blood collection from venous puncture and genetic analysis from each subject. The work conform to the Helsinki Declaration and was approved by the ethics committee of our hospital.

\subsection{TREM2 exon 2 sequencing}

Total genomic DNA was prepared from peripheral blood according to standard procedures. TREM2 exon 2 and at least 100 base pairs of its flanking introns were evaluated by polymerase chain reaction (PCR), the primers of which were designed to optimize denaturing high-performance liquid chromatography (dHPLC) conditions (forward primer: 5'-CACAGAGCAAGTGTTCAAAGC- $3^{\prime}$ and reverse primer: 5'-CACACAGACGCCCAAAAC-3' with the annealing temperature of $57^{\circ} \mathrm{C}$.

Preliminary dHPLC analysis was performed on the Wave nucleic acid fragment analysis system (Transgenomic, Santa Clara, CA USA), and samples with an altered dHPLC profile were sequenced. Nucleotide direct sequencing was performed on genomic DNA for both strands by ABI 3130xl DNA analyzer (Applied Biosystems, Foster City, CA, USA) and analyzed using SeqScape Software version 2.6 (Applied Biosystems, Foster City, CA, USA).

\subsection{Bioinformatic analyses}

A multiple protein alignment was constructed with multiple alignment by Bioedit (http://www.mbio.ncsu.edu/bioedit/bioedit. html) and CLUSTALW (http://clustalw.ddbj.nig.ac.jp/top-e.html) software.

The potential effect of the newly detected mutations on protein structure or function was analyzed with 4 prediction programs: PolyPhen2 (http://genetics.bwh.harvard.edu/pph2/), Pmut: (http://mmb.pcb.ub.es/PMut/), SNAP (http://rostlab.org/services/ snap/), and SIFT (http://sift.jcvi.org/www/SIFT_enst_submit.html).

\subsection{Statistical analysis}

Comparisons of each mutation and overall mutation frequencies between patients and HCs were compared by Pearson $\chi^{2}$ test. Logistic regression analysis was performed to assess odds ratio, and corresponding 95\% confidence intervals. Results are expressed as mean \pm SD or percentage, as reported. The significant level was established at $p<0.05$. Data analyses were carried out using SPSS 13.0 software (http://wwwspss.com).

\section{Results}

One thousand and thirty subjects were included in the present study, 352 patients with a diagnosis of FTD (aged $65.6 \pm 8.6$ years, $48.8 \%$ women), and, as control groups, $484 \mathrm{HCs}$ (aged $72.2 \pm 10.1$ years, 54.5\% women) and 194 patients with $\mathrm{AD}$ (aged $65.9 \pm 7.2$ years, $53.1 \%$ women).

In the overall sample, we found 8 genetic variations in 24 subjects in 24 subjects: Q33X, R47H, R62H, T66M, D87N, T96K, R98W, and S116C. As reported in Table 1, R47H, T66M, and S116C were found only in patients with FTD; Q33X was found in patients with both FTD and $\mathrm{AD}$, and $\mathrm{R} 62 \mathrm{H}, \mathrm{D} 87 \mathrm{~N}, \mathrm{~T} 96 \mathrm{~K}$, and R98W were also found in HCs. 
Table 2

In silico analyses of the amino acid changes found in the analyzed sample

\begin{tabular}{|c|c|c|c|c|c|c|c|c|}
\hline In silico software & Q33X & $\mathrm{R} 47 \mathrm{H}$ & $\mathrm{R} 62 \mathrm{H}$ & T66M & D87N & T96K & R98W & S116C \\
\hline PolyPhen2 (score ${ }^{\mathrm{a}}$ ) & Stop gained. NA & $\begin{array}{l}\text { Probably } \\
\text { damaging (1.0) }\end{array}$ & Benign (0.016) & $\begin{array}{l}\text { Probably } \\
\text { damaging (1.0) }\end{array}$ & $\begin{array}{l}\text { Probably } \\
\text { damaging (1.0) }\end{array}$ & $\begin{array}{l}\text { Probably } \\
\text { damaging (1.0) }\end{array}$ & $\begin{array}{l}\text { Probably } \\
\text { damaging (0.995) }\end{array}$ & $\begin{array}{l}\text { Probably } \\
\text { damaging (0.987) }\end{array}$ \\
\hline SNAP & Stop gained. NA & Nonneutral & Neutral & Nonneutral & Neutral & Nonneutral & Nonneutral & Neutral \\
\hline P-mut & Stop gained. NA & Pathologic & Pathologic & Pathologic & Neutral & Pathologic & Pathologic & Pathologic \\
\hline SIFT & Stop gained. NA & Tolerated & Tolerated & Damaging & Tolerated & Damaging & Damaging & Tolerated \\
\hline
\end{tabular}

Key: NA, not applicable.

a The lower the score, the more benign the substitution (range 0-1).

Overall, we found significantly more variants in exon 2 of TREM2 in patients with FTD than in HCs $(4.0 \%$ vs. $1.0 \%, p=0.005)$. Carriers of TREM2 exon 2 mutations had an almost 4-fold increased risk to develop FTD than noncarriers (odds ratio: 3.97, 95\% confidence intervals: $1.42-11.12$ ). In FTD, 6 of 14 patients carried heterozygous TREM2 mutations that were not found in HCs. As reported in Table 2, in silico analysis demonstrated that the Q33X variant (cytosine to thymine change in position 6818), previously associated with Nasu-Hakola disease in the homozygous state, resulting in a TAG stop codon with a loss of function of the TREM2 protein. The $\mathrm{R} 47 \mathrm{H}$ mutation was characterized by a single point mutation in the CGC codon, with the substitution of guanine with adenine, and was predicted to be damaging in 3 of 4 in silico analyses. The T66M mutation was characterized by a single point mutation in the ACG codon, resulting in a replacement of threonine at position 66 with methionine; all in silico analyses predicted a pathologic effect of this substitution, and this mutation was previously associated with Nasu-Hakola disease in homozygosity. S116C has not been previously described in the literature and causes a substitution of adenine to thymine; no clear-cut results were obtained by in silico analysis because 2 of 4 prediction analyses suggested neutral effect.

In Table 3, demographic and clinical characteristics of patients with FTD carrying TREM2 Q33X, R47H, T66M, and S116C are reported. In most of these cases, patients presented an early age of onset (range: $52-68$ years), and $66.7 \%$ were women. Family history was unremarkable in 5 of 6 patients. Half had a diagnosis of svPPA (2 Q33X, 1 S116C), and the others had a diagnosis of bvFTD (1 Q33X, $1 \mathrm{R} 47 \mathrm{H}$, and $1 \mathrm{~T} 66 \mathrm{M})$, with a phenotypic variability in patients carrying the same genetic variation.

When the AD control group was considered to elucidate whether FTD and AD may share a common at-risk genetic background, no significant differences in the overall number of TREM2 exon 2 mutations were found ( $4.0 \%$ vs. 2.6, $p=0.46$ ). Notably, in $1.5 \%$ of patients with $\operatorname{AD}(\mathrm{n}=3,2$ women, age range $58-69$ years), a heterozygous Q33X mutation was found, whereas 1 patient carried $\mathrm{R} 62 \mathrm{H}$ and 1 carried R98W, both of which were also found in HCs.

Finally, when the AD group was compared with the HC group, we found significantly more Q33X variants of TREM2 in the former group $(\mathrm{n}=3,1.5 \%)$ then the latter $(0.0 \%, p=0.023)$. Patients with $A D$ carrying Q33X mutation showed late-onset disease (aged 65-75 years), 2 of 3 were women, and none carried the apolipoprotein $\mathrm{E} \varepsilon 4$ allele.

Moreover, in $\mathrm{AD}$, we found $\mathrm{R} 62 \mathrm{H}$ and R98W mutations, which were detected in HCs as well. We did not find any difference in overall frequency of mutations in patients with $A D$ versus HCs, presumably because of the small sample of patients with AD.

\section{Discussion}

TREM2 gene has recently gained great interest in the dementia field, as the identification of homozygous mutations in cases of FTDlike phenotype (Guerreiro et al., 2013a, 2013c), and the demonstration that TREM2 exon 2 genetic variations are risk factors for AD in heterozygosis (Guerreiro et al., 2013b; Jonsson et al., 2013). With these premises, we hypothesized that heterozygous TREM2 mutations might be related to FTD risk as well. In the present work, we screened a large sample of patients and HCs for TREM2 exon 2 genetic variations and found that FTD patients showed increased frequency of heterozygous TREM2 mutations compared with HCs. Interestingly, we confirmed that Q33X, R47H, and T66M mutations were significantly associated with the disease and absent in healthy subjects. Q33X and T66M mutations have previously been demonstrated to cause Nasu-Hakola disease in the homozygous state (Paloneva et al., 2000, 2002), and the heterozygous R47H mutation has been associated with AD risk (Guerreiro et al., 2013b; Jonsson et al., 2013). Accordingly, in silico analysis of these mutations suggested a loss of function in TREM2; this might suggest that these variants are causal mutations, even in heterozygosis, rather than risk factors.

Notably, different clinical phenotypes were observed in FTD patients carrying these TREM2 genetic variations with variable age at disease onset and inconsistent family history for neurodegenerative disorders. Interestingly, patients presented either bvFTD or svPPA, and this is the first association study suggesting a genetic risk factor for svPPA phenotype.

Recently studies on TREM2 genetic variations have been published in France (Lattante et al., 2013), the United States (Rayaprolu et al., 2013), and Colombia (Giraldo et al., 2013) with different mutation frequencies. No work exploring the role of TREM2 in the Italian population is available yet.

TREM2 encodes a single-pass type I membrane protein that forms a receptor-signaling complex with the TYRO protein tyrosine kinase-binding protein (TYROBP) that is involved in the activation of immune responses in immune cells, as macrophages and dendritic cells. It is involved in chronic inflammation by triggering the production of constitutive rather than inflammatory chemokines and cytokines and has a critical role in microglia clearance (Neumann et al., 2013; Paloneva et al., 2003; Takahashi et al., 2005).

Interestingly, we also found TREM2 mutations in our AD patients, confirming previous data (Guerreito et al., 2013b; Jonsson et al., 2013) and suggesting a common pathway in these 2 neurologic disorders, likely associated to inflammation processes. Indeed, emerging evidence has underlined a greater interest in mechanisms targeting inflammation in both diseases (Galimberti and Scarpini, 2011; Piguet, 2013). Furthermore, in human control brain, TREM2 was expressed at higher levels in the hippocampus and neocortex (Guerreiro et al., 2013a), and this pattern of

Table 3

Demographic and clinical characteristics of FTD patients carrying TREM2 exon 2 mutations

\begin{tabular}{lllll}
\hline & Age at onset & Gender & Family history & Phenotype \\
\hline ID1 Q33X & 68 & F & No & svPPA \\
ID2 Q33X & 52 & F & No & svPPA \\
ID3 Q33X & 53 & F & Yes & bvFTD \\
ID4 R47H & NA & M & No & bvFTD \\
ID5 T66M & 55 & M & No & bvFTD \\
ID6 S116C & 59 & F & No & svPPA \\
\hline
\end{tabular}

Key: bvFTD, behavioral variant of primary progressive aphasia; NA, not available; SvPPA, semantic variant of primary progressive aphasia. 
expression is partly consistent with pathologic features observed in both FTD and AD.

The effect of TREM2 genetic variations in FTD cases and the possible interaction with protein involved in the known pathogenetic mechanisms of the disease remains to be elucidated. Functional activity assays will be required to determine how these identified mutations might compromise TREM2 function, providing new clues to the mechanisms underlying the pathogenesis of FTD. Unfortunately, we were not able to assess the effect of TREM2 genetic variation in our patients because it is expressed only in microglia and not in circulating monocytes.

This is a preliminary study, and we do not exclude the possibility that the frequency of heterozygous mutations we found is partially due to the relatively high number of cases $(n=3)$ with Nasu-Hakola disease (i.e., homozygous Q33X mutation) that we diagnosed in our geographic area (Bock et al., 2013). Moreover, it is reasonable to suppose that other mutations in TREM2, beyond exon 2, may be identified. However, as previously suggested (Guerreiro et al., 2013b), it may be that rare variants in the heterozygous state may be risk factors for late-onset complex disorders, determining defined early-onset disease when in homozygosis.

In conclusion, we report that TREM2 variants might be considered risk variants in FTD not only in homozygosis as previously demonstrated but in heterozygosis as well. Moreover, the absence of certain variants, in both our control population and others previously published (Guerreiro et al., 2013b; Jonsson et al., 2013; Paloeva et al., 2001), raises the question of whether such variants are mutations leading to dementia and Nasu-Hakola disease rather than polymorphisms influencing the risk of developing FTD. Whether TREM2 is a major gene or a modifier gene for FTD and AD remains to be determined. Future confirmatory clinical and experimental studies are needed to elucidate the role of this gene in FTD.

\section{Disclosure statement}

The authors have no actual or potential conflicts of interest.

\section{Acknowledgements}

This work was supported by grants from the Italian Ministry of Health (Ricerca Corrente), Fondazione Monzino, Cassa di Risparmio di Pistoia e Pescia (grant 2012), and the Cassa di Risparmio di Firenze (grant 2012).The authors thank Drs Andrea Pilotto and Alberto Benussi for editing the manuscript.

\section{References}

Bock, V., Botturi, A., Gaviani, P., Lamperti, E., Maccagnano, C., Piccio, L., Silvani, A., Salmaggi, A., 2013. Polycystic lipomembranous osteodysplasia with sclerosing leukoencephalopathy (PLOSL): a new report of an Italian woman and review of the literature. J. Neurol. Sci. 326, 115-119.

Borroni, B., Bonvicini, C., Galimberti, D., Tremolizzo, L., Papetti, A., Archetti, S., Turla, M., Alberici, A., Agosti, C., Premi, E., Appollonio, I., Rainero, I., Ferrarese, C., Gennarelli, M., Scarpini, E., Padovani, A., 2011. Founder effect and estimation of the age of the progranulin Thr272fs mutation in 14 Italian pedigrees with frontotemporal lobar degeneration. Neurobiol. Aging 32 (3), 555.e1-555.e8.

Galimberti, D., Fenoglio, C., Serpente, M. Villa, C., Bonsi, R. Arighi, A. Fumagalli, G.G., Del Bo, R., Bruni, A.C., Anfossi, M., Clodomiro, A., Cupidi, C., Nacmias, B., Sorbi, S., Piaceri, I., Bagnoli, S., Bessi, V., Marcone, A., Cerami, C., Cappa, S.F., Filippi, M., Agosta, F., Magnani, G., Comi, G., Franceschi, M., Rainero, I., Giordana, M., Rubino, E., Ferrero, P., Rogaeva, E., Xi, Z., Confaloni, A., Piscopo, P., Bruno, G., Talarico, G., Cagnin, A., Clerici, F., Dell'Osso, B., Comi, G.P., Altamura, A.C., Mariani, C., Scarpini, E., 2013. Autosomal dominant frontotemporal lobar degeneration due to the C9ORF72 hexanucleotide repeat expansion: late-onset psychotic clinical presentation. Biol. Psychiatry 74, $384-391$.

Galimberti, D., Scarpini, E., 2011. Inflammation and oxidative damage in Alzheimer's disease: friend or foe? Front. Biosci. (Schol. Ed.) 3, 252-266.

Giraldo, M., Lopera, F., Siniard, A.L., Corneveaux, J.J., Schrauwen, I., Carvajal, J., Muñoz, C., Ramirez-Restrepo, M., Gaiteri, C., Myers, A.J., Caselli, R.J., Kosik, K.S.,
Reiman, E.M., Huentelman, M.J., 2013. Variants in triggering receptor expressed on myeloid cells 2 are associated with both behavioral variant frontotemporal lobar degeneration and Alzheimer's disease. Neurobiol. Aging 34 2077.e11-2077.e18.

Gorno-Tempini, M.L., Hillis, A.E., Weintraub, S., Kertesz, A., Mendez, M., Cappa, S.F. Ogar, J.M., Rohrer, J.D., Black, S., Boeve, B.F., Manes, F., Dronkers, N.F., Vandenberghe, R., Rascovsky, K., Patterson, K., Miller, B.L., Knopman, D.S. Hodges, J.R., Mesulam, M.M., Grossman, M., 2011. Classification of primary progressive aphasia and its variants. Neurology 76, 1006-1014.

Guerreiro, R.J., Lohmann, E., Brás, J.M., Gibbs, J.R., Rohrer, J.D., Gurunlian, N. Dursun, B., Bilgic, B., Hanagasi, H., Gurvit, H., Emre, M., Singleton, A., Hardy, J., 2013a. Using exome sequencing to reveal mutations in TREM2 presenting as a frontotemporal dementia-like syndrome without bone involvement. JAMA Neurol. 70, 78-84.

Guerreiro, R., Wojtas, A., Bras, J., Carrasquillo, M., Rogaeva, E., Majounie, E. Cruchaga, C., Sassi, C., Kauwe, J.S., Younkin, S., Hazrati, L., Collinge, J., Pocock, J., Lashley, T., Williams, J., Lambert, J.C., Amouyel, P., Goate, A., Rademakers, R. Morgan, K., Powell, J., St George-Hyslop, P., Singleton, A., Hardy, J., Alzheimer Genetic Analysis Group, 2013b. TREM2 variants in Alzheimer's disease. N. Engl. J. Med. 368, 117-127.

Guerreiro, R., Bilgic, B., Guven, G., Brás, J., Rohrer, J., Lohmann, E., Hanagasi, H., Gurvit, H., Emre, M., 2013c. A novel compound heterozygous mutation in TREM2 found in a Turkish frontotemporal dementia-like family. Neurobiol. Aging 34, 2890.e1-2890.e5.

Jonsson, T., Stefansson, H., Steinberg, S., Jonsdottir, I., Jonsson, P.V., Snaedal, J., Bjornsson, S., Huttenlocher, J., Levey, A.I., Lah, J.J., Rujescu, D., Hampel, H. Giegling, I., Andreassen, O.A., Engedal, K., Ulstein, I., Djurovic, S., IbrahimVerbaas, C., Hofman, A., Ikram, M.A., van Duijn, C.M., Thorsteinsdottir, U., Kong, A., Stefansson, K., 2013. Variant of TREM2 associated with the risk of Alzheimer's disease. N. Engl. J. Med. 368, 107-116.

Lattante, S., Le Ber, I., Camuzat, A., Dayan, S., Godard, C., Van Bortel, I., De Septenville, A., Ciura, S., Brice, A., Kabashi, E., French Research Network on FTD and FTD-ALS, 2013. TREM2 mutations are rare in a French cohort of patients with frontotemporal dementia. Neurobiol. Aging 34, 2443.e1-2443.e2.

McKhann, G., Drachman, D., Folstein, M., Katzman, R., Price, D., Stadlan, E.M., 1984 Clinical diagnosis of Alzheimer's disease: report of the NIN CDS-ADRDA Work Group under the auspices of Department of Health and Human Services Tasks Force on Alzheimer's Disease. Neurology 34, 939-944.

Neumann, H., Daly, M.J., 2013. Variant TREM2 as risk factor for Alzheimer's disease. N. Engl. J. Med. 368, 182-184.

Paloeva, J., Autti, T., Raininko, R., Partanen, J., Salonen, O., Puranen, M., Hakola, P., Haltia, M., 2001. CNS manifestations of Nasu-Hakola disease: a frontal dementia with bone cysts. Neurology 56, 1552-1558.

Paloneva, J., Kestilä, M., Wu, J., Salminen, A., Bohling, T., Ruotsalainen, V., Hakola, P., Bakker, A.B., Phillips, J.H., Pekkarinen, P., Lanier, L.L., Timonen, T., Peltonen, L. 2000. Loss-of-function mutations in TYROBP (DAP12) result in a presenile dementia with bone cysts. Nat. Genet. 25, 357-361.

Paloneva, J., Manninen, T., Christman, G., Hovanes, K., Mandelin, J., Adolfsson, R., Bianchin, M., Bird, T., Miranda, R., Salmaggi, A., Tranebjaerg, L., Konttinen, Y. Peltonen, L., 2002. Mutations in two genes encoding different subunits of a receptor signaling complex result in an identical disease phenotype. Am. J. Hum. Genet. 71, 656-662.

Paloneva, J., Mandelin, J., Kiialainen, A., Bohling, T., Prudlo, J., Hakola, P., Haltia, M., Konttinen, Y.T., Peltonen, L., 2003. DAP12/TREM2 deficiency results in impaired osteoclast differentiation and osteoporotic features. J. Exp. Med. 198, 669-675.

Piguet, O., 2013. Neurodegenerative disease: frontotemporal dementia-time to target inflammation? Nat. Rev. Neurol 9, 304-305.

Rademakers, R., Neumann, M., Mackenzie, I.R., 2012. Advances in understanding the molecular basis of frontotemporal dementia. Nat. Rev. Neurol 8, 423-434.

Rayaprolu, S., Mullen, B., Baker, M., Lynch, T., Finger, E., Seeley, W.W., Hatanpaa, K.J. Lomen-Hoerth, C., Kertesz, A., Bigio, E.H., Lippa, C., Josephs, K.A., Knopman, D.S. White 3rd, C.L., Caselli, R., Mackenzie, I.R., Miller, B.L., Boczarska-Jedynak, M. Opala, G., Krygowska-Wajs, A., Barcikowska, M., Younkin, S.G., Petersen, R.C., Ertekin-Taner, N., Uitti, R.J., Meschia, J.F., Boylan, K.B., Boeve, B.F., GraffRadford, N.R., Wszolek, Z.K., Dickson, D.W., Rademaker, R., Ross, O.A., 2013. TREM2 in neurodegeneration: evidence for association of the p.R47H variant with frontotemporal dementia and Parkinson's disease. Mol. Neurodegener. 8, 19

Rascovsky, K., Hodges, J.R., Knopman, D., Mendez, M.F., Kramer, J.H., Neuhaus, J., van Swieten, J.C., Seelaar, H., Dopper, E.G., Onyike, C.U., Hillis, A.E., Josephs, K.A., Boeve, B.F., Kertesz, A., Seeley, W.W., Rankin, K.P., Johnson, J.K., GornoTempini, M.L., Rosen, H., Prioleau-Latham, C.E., Lee, A., Kipps, C.M., Lillo, P., Piguet, O., Rohrer, J.D., Rossor, M.N., Warren, J.D., Fox, N.C., Galasko, D Salmon, D.P., Black, S.E., Mesulam, M., Weintraub, S., Dickerson, B.C., DiehlSchmid, J., Pasquier, F., Deramecourt, V., Lebert, F., Pijnenburg, Y., Chow, T.W. Manes, F., Grafman, J., Cappa, S.F., Freedman, M., Grossman, M., Miller, B.L., 2011 Sensitivity of revised diagnostic criteria for the behavioural variant of frontotemporal dementia. Brain 134, 2456-2477.

Rohrer, J.D., Warren, J.D., 2011. Phenotypic signatures of genetic frontotempora dementia. Curr. Opin. Neurol. 24, 542-549.

Seelaar, H., Rohrer, J.D., Pijnenburg, Y.A.L., Fox, N.C., Van Swieten, J.C., 2011. Clinical, genetic and pathological heterogeneity of frontotemporal dementia: a review. JNNP 82, 476-486.

Takahashi, K., Rochford, C.D., Neumann, H., 2005. Clearance of apoptotic neurons without inflammation by microglial triggering receptor expressed on myeloid cells-2. J. Exp. Med. 201, 647-657. 